\title{
II. Ueber ein neues 0cularmikrometer und dessen Anwendung in der mikroskopischen Krystallographie.
}

\author{
Von
}

S. Krysiński in Dorpat.

Der Erste, welcher das Mikroskop zur Messung der diëdrischen Winkel mikroskopischer Krystalle benutzte, war G. Wertheim. In seiner Abhandlung*) beschreibt er zuerst die von ihm projectirte Messungsmethode und discutirt nachträglich die Abhängigkeit ibrer Genauigkeit von der Schärfe der Einstellung des Mikroskops. Seine Methode gründet sich darauf, dass man den Neigungswinkel zweier Ebenen leicht ausrechnen kann, wenn man die Lage von sechs Punkten im Raume bestimmt, von denen je drei auf einer dieser Ebenen, aber nicht alle drei in einer geraden Linie liegen $\left.{ }^{* *}\right)$.

*) Ueber eine am zusammengesetzten Mikroskop angebrachte Vorrichtung zum $Z$ wecke der Messung in der Tieferichtung und eine hierauf gegründete neue Methode der Krystallbestimmung. Sitzungsberichte der Mathematisch-Naturwissenschaftlichen Classe der kaiserlichen Akademie der Wissenschaften. Wien 1862, 45, 157.

**) In der Beurtheilung der Genauigkeit der vorgeschlagenen Messungsmethode geht Wertheim von der Bestimmung des wahrscheinlichen Fehlers bei der möglichst scharfen Einstellung des Mikroskops aus, und giebt an, dass für das von ihm benutzte (1862! der Verfertiger des Mikroskops ist nicht angegeben) der Einstellungsfehler (der maximale Werth der Unsicherheit bei der möglichst scharfen Einstellung als Mittelwertb aus zelın Messungen bestimmt) für seine 70 fache Vergrösserung (Oc. 1, Syst. I) $0,011 \mathrm{~mm}$ und für seine 280 fache (0c. 1, Syst. V) $0,00075 \mathrm{~mm}$ nicht erreichen soll. Diese Unsicherheitswerthe benutzend, discutirt Wertheim die Grösse des wahrseheinlichen Fehlers bei der Winkelbestimmung. Seine mathematische Deduction bezieht sich sichtlich aber nur auf den sehr speciellen Fall, in welchem die Kante des diëdrischen Winkels zur Tischebene des Mikroskopes genau parallel wäre, und die zwei auf den Krystalllächen entsprechend gewählten Punkte mit dem auf der Kante gewählten auf einer zu dieser Kante senkrechten Ebene liegen würden. Nach ganz willkürlichen Vernachlässigungen der

Groth, Zeitschrift f. Krystallogr. XIV: 
Um die rechtwinkligen Coordinaten der gewählten Punkle zu messen, benutzt Wertheim: 1) ein Fadenkreuzocular, 2) eine feine Theilung am Kopfe der Mikrometerschraube des Mikroskops und endlich 3) einen nach zwei zu einander senkrechten Richtungen vermittelst Schrauben beweglichen Objecttisch.

Mit diesen Hilfsmitteln wird die Messung in folgender Weise ausgeführt :

Nachdem auf die gewöhnliche Tischplatte des Mikroskops der bewegliche Objecttisch aufgelegt und befestigt ist, legt man auf denselben den Objectträger sammt dem Präparat, beschaut dasselbe sorgfăltig, wählt einen entsprechenden Krystall aus, centrirt ihn annäbernd (durch freie Handverschiebung des Objectträgers), wählt auf zwei gut ausgebildeten Flächen desselben je drei leicht zu erkennende Punkte aus, befestigt den Objeclträger auf dem beweglichen Objecttische und notirt den Stand der Indices auf den Schraubenköpfen des beweglichen Tisches und der Mikrometerschraube des Mikroskops. Dann stellt man das Mikroskop mittelst der Mikrometerschraube auf einen der gewählten Punkte ein und verschiebt durch Drebung der einen Objecttischschraube das Präparat so lange, bis der gewählte Punkt zur genauen Coincidenz mit dem zur Bewegungsrichtung senkrechten Fadenkreuzarm gebracht wird. Durch Drebung der zweiten Objecttischschraube wird dann der gewäblle Punkt zur Coincidenz auch mit dem zweilen Fadenkreuzarm gebracht, also genau auf ihren Kreuzungspunkt gestellt. Endlich wird das Mikroskop nochmals möglichst scharf auf den jetzt centrirten Punkt eingestellt und der Stand aller Indices nochmals ahgelesen.

Wenn man nun die Richtung des Fadenkreuzarmes, der von links nach rechts geht, als die $X$-Axe, die des von vorn nach hinten gebenden Armes als die $Y$-Axe, das Bild ibres Kreuzungspunktes als den Coordinatenanfang, die durch diesen Punkt zu den zwei ersten Richtungen gezogene Senkrechte (also parallel zu der mit der Mikrometerschraube dem Tubus mitgetheilten Bewegungsrichtung) als die $Z$-Axe betrachtet und den absoluten Werth einer Theilung der Indices kennt, so hat man dadurch alle drei auf die genannten Axen bezogenen Coordinaten des gewählten Punktes bestimmt.

durchaus nicht verschwindenden kleinen Glieder des Zählers und Nenners in der gefundenen mathematischen Formel findet endlich Wertheim, dass für einen Krystall, auf welchem die Entfernungen der auf den Flächen liegenden Punkte von den auf der Kante liegenden $0,5 \mathrm{~mm}$ betragen könnten, der wahrscheinliche Winkelbestimmungsfehler für seine 70 fache Vergrösserung $3^{0} 3^{\prime}$, für seine 280 fache $14^{\prime}$ (Bogenminuten) betragen würden. Für einen Krystall, auf dem die entsprechenden Entfernungen $1 \mathrm{~mm}$ betragen würden, berechnet er den Fehler auf 104 $7^{\prime}$ entsprechend 7 ' (Bogenminuten).

Die Prämissen und die Art der Deduction der gefundenen Fehlergrenzen schliessen, wie ich meine, eine weitere Erörterung derselben aus. 
Ganz ähnlich verfährt man selbstverständlich, um die Coordinaten der übrigen Punkte zu bestimmen.

Die soeben beschriebene Coordinatenmessungsmethode leidet hauptsächlich an dem Mangel, dass die Flächencoordinaten $X$ und $Y$ nicht genau genug bestimmt werden können. Diese Coordinaten werden nämlich gemessen durch Drehung der an dem beweglichen Objecttische angebrachten Schrauben. Eine Schraube aber, die eine Einhundertstel-Millimeter-Verschiebung genau auszuführen und abzulesen gestattet, muss mit einer ausserordentlichen Präcision ausgeführt werden und lässt sich nur schwer an dem beweglichen Objecttische anbringen. Die an den jetzt gangbaren Objecttischen angebrachten Schrauben gehören zu den allergewöhnlichsten und gestatten demzufolge auch absolut keine Präcision bei der Messung.

Der bekannte Jenenser Optiker $\mathrm{Z}$ e is s liefert allerdings ein Objecttischschraubenmikrometer, welches die Verschiebung um 0,002 mm direct abzulesen gestattet. Mit Hille dieses complicirten Instrumentes lässt sich jedoch die Verscbiebung nur in einer Richtung ausführen. Sollte, wie es für die Wertheim'sche Methode unentbehrlich ist, die Verschiebung in zwei Richtungen erfolgen, so würde das sonst schon recht complicirte und theure Instrument (120 Mark) noch complicirter, noch theurer und sicber nicht präciser werden.

Die ganze Schwierigkeit aber würde mit einem Schlage beseitigt, wenn man die Schrauben nur zur Ausführung der Bewegung, nicht aber gleichzeitig zur Messung derselben benutzen wlirde, und sie statt an dem Objectlische, am Ocular befestigen würde.

Im Anfang des Jahres 1882 hat Herr Dr. Hartnack in Polsdam die Liebenswürdigkeit gehabt, das von ihm unlängst construirle sogenannte nNeue bewegliche Ocularmikrometer « auf mein Ansuchen und nach meinem Vorschlag zu reconstruiren. Fur die specielle, uns hier interessirende Bestimmung babe ich vor Kurzem an demselben Ocular eine neue Veränderung angebracht. Nach dieser letzten Veränderung sieht das Instrument, dessen constructive Details Herr Hartnack in der "Zeitschrift für Instrumentenkunde « demnächst beschreiben wird, etwa folgendermassen aus.

Auf dem gewöhnlichen Ocularcylinder von etwa $22 \mathrm{~mm}$ Durchmesser ist in der Höhe von etwa $12 \mathrm{~mm}$ vom unteren Ende gerechnet eine Metalltrommel von etwa $55 \mathrm{~mm}$ Durchmesser und $10 \mathrm{~mm}$ Höhe befestigt. Diese Trommel besteht aus zwei in einander rotirenden Gylindern, von denen der untere fest mit dem unteren Ende des Oculars verbunden ist, während der obere, ebenfalls fest mit dem oberen Theile des Oculars verbundene, sanft in dem unteren Theile drehbar ist. In dem unteren Theile ist in einem gewöhnlichen Rahmen das Fadenkreuz befestigt, im oberen, dicht über dem Fadenkreuz, die bewegliche Mikrometerscala.

Die Mikrometerscala, welche ebenso wie das Fadenkreuz auf einem 
Glasplättchen photographirt ist, lässt sich vermittelst einer seitlich über der Trommel hervorragenden Schraube leicht und sicher nebst dem sie tragenden Rahmen in einer Coulisse hin und her bewegen. Diese Coulisse aber ist selbst in einer anderen Coulisse befestigt, in der sie, in einer zu der ersten genau senkrechten Richtung ebenfalls mittelst einer nach aussen hervorragenden Schraube sicher bewegt werden kann.

Die auf einem Glasplättchen photographirte und deutlich bezifferte Scala besteht aus einem rechtwinkJigen Dreieck, dessen eine Kathete genau zehnmal so lang ist als die andere. Die lange Kathete ist in hundert gleiche Theile getheilt und die zu ihr selbstverständlich senkrechten Theilungsstriche haben alle mit Ausnahme der Fünfer- und Zehnerstriche, welche wie ublich noch länger sind, die Länge der kurzen Kathete.

Aus dieser Construction folgt, dass die zwischen der Hypotenuse und der langen Kathete liegenden Abschnitte der Theilungsstriche genau dem zehnten Theile der entsprechenden Abschnitte der langen Kathete gleich sind, dass also z. B. der zwischen der Hypotenuse und der langen Kathete liegende Theil des bei der Zahl ö7 stehenden Theilungsstriches genau 5,7 Theilstriche lang ist.

Durch einen unter der Trommel angebrachten Stift und einen entsprechenden Schlitz im oberen Tubusrande des Mikroskops ist für die Unbeweglichkeit des unteren Trommelcylinders mit dem Fadenkreuz genügend gesorgt. Durch zwei an beiden Trommeltheilen angebrachte Indices und Schnappfeder ist auch Sorge dafür getragen, dass man obne Weileres die lange Kathete der Scala in eine parallele oder senkrechte Richtung zu einem Fadenkreuzarme bringen kann. Es befindet sich endlich auf der Perjpherie des unteren Trommelcylinders eine Gradtheilung eingravirt und ibr gegenüber auf der Peripherie des oberen beweglichen Trommelcylinders ein entsprechender Nonius.

Die Augenlinse dieses Ocularmikrometers ist wie ublich zum Heben und Senken eingerichtet, damit man das Bild der Scala auf die Entfernung der deutlichen Sehweite des Beobachters bringen kann, und da die Entfernung zwischen dem Photogramm der Scala und dem des Fadenk reuzes nur minimal ist, lässt sich das Bild beider gleichzeilig gleich deutlich sehen.

Die Messung der mikroskopischen Gegenstände, deren Grösse den Werth von zehn Theilstrichen der Scala nicht ubersteigt, wird mit diesem Instrument so vorgenommen, dass man zuerst durch entsprechende Drehung des oberen Theiles des Oculars und der Trommelschrauben die lange Kathete der Scala zur genauen Berührung mit einem Rande (eventuell Ende) des Gegenstandes bringt, dann aber wird die Scala durch die Drehung der zu der langen Katbete parallelen Schraube so weit vorgeschoben, bis ihre Hypotenuse das gemessene Object im diametral entgegengesetzten Punkte (Rande, Ende) berührt. Da aber bei dieser letzten Verschiebung die lange 
Kathete stets in ihrer ursprünglichen Richtung verbleibt, wird sie auch stets das Object im selben Punkte berühren, und in Folge dessen wird der durch den Beruhrungspunkt der Hypotenuse mit dem zweiten Ende des Objectes gehende Theilungsstrich mit dem gewählten Durchmesser des Objectes gänzlich coincidiren und seine Länge direct angeben,

Da die Scala durch die beiden Schrauben uber das ganze Sehfeld gefuhrt und durch eine entsprechende Drehung in jede beliebige Neigung zu dem unbeweglichen Fadenkreuz gebracht werden kann, ist die beschriebene Art der Messung, die wir im Folgenden kurz »Einkeilung ( nennen werden, auf jedem beliebigen Punkte des Sehfeldes gleich leicht ausführbar.

Wenn aber der gewählte Durchmesser des Objectes grösser als zehn Theilstriche der Scala wäre, also nicht mehr eingekeilt werden könnte, so braucht man nur durch entsprechende Drehung am oberen Theile des Oculars und an den Trommelschrauben die lange Kathete zur Coincidenz mit demselben zu bringen, dann einen der Scala-Theilungstriche genau mit einem Ende des Durchmessers zu accordiren, um dessen Länge direct abzulesen.

So wie bei jedem anderen Ocularmikrometer muss man auch bei dem beschriebenen Instrument für jedes Objectivsystem den Werth einer Scalatheilung mit der grössten Genauigkeit bestimmen. Dieses geschieht wie bekannt folgendermassen:

Als Präparat legt man auf den Objecttisch ein Objectmikrometer, d. i. einen Glasstreifen, auf dem ein Millimeter in hundert Theile getheilt ist, stellt das Mikroskop auf die Theilung ein, bringt durch entsprechende Drebung des oberen Oculartheiles und der an der Trommel angebrachten Schraube die kurze Kathete der Scala zur Coincidenz mit einem Theilungsstriche des Objectmikrometers und bestimmt, zwischen welchen Zahlen der Theilungsstriche des Object-und Ocularmikrometers die grösste Uebereinstimmung stattfindet. Hat man z. B. gefunden, dass 88 Theilstriche des Ocularmikrometers genau 20 Theilstrichen des Objectmikrometers entsprechen, so muss man noch prufen, ob dieselbe Uebereinslimmung auch bei den aliquoten Theilen und dem Vielfachen dieser Zablen stattfindet, ob also 44, 22 u. s. w. Theilstriche des Oeularmikrometers 10, 5 u. s. w. Theilstrichen des Objectmikrometers entsprechen. Ist das der Fall, so kann man das Maass als genau betrachten, und wenn z. B. wie in unserem Fall 22 Theile des Ocularmikrometers 5 Theilen des Objectmikrometers, also $0,55 \mathrm{~mm}$, entsprechen, so entspricht einem Theilstriche des Ocularmikrometers, wie man sich durch einfache Division überzeugen kann, 0,002254 mm oder 2,254 $\mu$ (Mikromillimeter).

Wenn aber eise gänzli ch e Uebereinstimmung úberhaupt nicht vorhanden wäre, so hat man die Zahlen der Theilstriche des Ocular- und Objectmikrometers zu nehmen, zwischen welchen die grös s te Uebereinstimmung herrscht und dabei immer den grösstmöglichen Zahlen den Vorzug 
gehen. Wenn z. B. sechs Theilstriche des Ocularmikrometers nur etwas länger als fünf Theilstriche des Objectmikrometers wären, darf man, wenn auch der Fehler nur ein minimaler sein sollte, nicht diese Zahlen der Rechnung zu Grunde legen, sondern bestimmen, wie vielen Theilen des Objectmikrometers 96 also $16 \times 6$ Theile des Ocularmikrometers entsprechen, und wenn wir finden, dass 96 Theile des Ocularmikrometers zwischen 81 und 82 des Objectmikrometers enthalten sind, dass

96 Th. d. Oc.-M. $>81$ Th. d. Obj.-M.

96 Th. d. Oc.-M. $<82$ Th. d. Obj.-M. und

96 Th. d. Oc.-M. fast $=81 \frac{1}{2}$ Th. d. Obj.-M.,

sind wir s i cher, dass

1 Th. d. Oc. - M. $>8,438 \mu$

1 Th. d. Oc.-M. $<8,541 \mu$ und vermuthen dass

1 Th. d. Oc.-M. fasl $=8,489$.

Wenn wir aber von der ersten Zahl

$$
6 \text { Th. d. Oc.-M. fast }=5 \text { Th. d. Obj.-M. }
$$

ausgehen wollten, bei der uns die Uebereinstimmung die grösste schien, würden wir erhalten, dass

$$
\text { 1. Th. d. Oc.-M. fast }=8,333 \mu \text {, }
$$

das ist einer unstreitig zu kleinen Zahl, da sicher

$$
1 \text { Th. d. Oc.-M. }>8,438 \mu \text {. }
$$

Diese Arbeit muss man unbedingt selbst vornehmen, um sich eine Werthtabelle der Mikrometertheilung für jedes Objectivsystem berzustellen. Auf die von den Optikern zu den Mikroskopen beigegebenen Tabellen kann man sich durchaus nicht verlassen, weil in den letzteren Unterschiede von $50-100 \%$ durchaus nicbt zu den Ausnahmen gehören.

Die Flächencoordinaten $X$ und $Y$ eines Punktes im Raume lassen sich mit Hilfe des beschriebenen Instrumentes ebenso leicht wie genau bestimmen.

Zu diesem Zwecke braucht man nur:

A. Wenn die Entfernung des gewählten Punktes von den Coordinatenaxen nicht über zehn Theilstriche der Scala beträgt, selbe zweimal einzukeilen, was man auf folgende Weise bewerkstelligt: durch Drehung des oberen Trommeltheiles und der entsprechenden Trommelschraube bringt man die lange Kathete zur genauen Coincidenz mit der $X$-Axe, und durch Drehung der zu der langen Katbete parallelen Schraube verschiebt man dann die Scala, bis die Hypotenuse den gewählten Punkt beruhrt. Da bei dieser Verschiebung die lange Kathete immer auf der $X$-Axe verbleibt, giebt die Länge des durch den gewählten Punkt gehenden Theilungsstrichabschnittes die senkrechte Entfernung des gegebenen Punktes von der 
X-Axe, also die Y-Coordinate direct an. Durch neue Drehung um 90 Grad wird dann die lange Kathete zur Coincidenz mit der $Y$-Axe gebracht und durch Drehung der entsprechenden Schraube wird die Scala soweit verschoben, bis die Hypotenuse abermals durch den gewählten Punkt geht, und endlich wird noch die Länge der auf diese Weise gefundenen $X$-Coordinate direct abgelesen.

B. Wenn die Entfernung des gewählten Punktes von den Axen grösser als zehn Theilstriche der Scala wäre, so müsste man selbstverständlich auf das Einkeilen verzichten und die Messung wie folgt ausfuhren.

1) Durch Drehung des oberen Oculartheiles muss man die lange Kathete zur Coincidenz mit der $X$-Axe bringen.

2) Durch Drehung der entsprechenden Trommelschraube wird die Scala parallel zu ibrer erslen Lage soweit verschoben, bis die lange Kathete durch den gewählten Punkt geht.

Da aber in Folge der beschriebenen Construction des Instrumentes die lange Kathete bei dieser Verschiebung stets parallel zu ihrer ersten Lage (zur $X$-Axe), also senkrecht auf die $Y$-Axe verbleibt, kann man auf ibr unmittelbar die senkrechte Entfernung des gewählten Punktes von der $Y$ Axe, also seine $X$-Coordinate ablesen.

Durch ein ganz entsprechendes Verfahren wurde man auch die zweite Coordinate bestimmen müssen.

Die Genauigkeit der beschriebenen Messungsmethode hängt in erster Linie von der Genauigkeit der Scala selbst und der Präcision der Ausführung und Richtung der Coulissen ab. Die Erfüllung dieses Postualates macht aber glucklicher Weise keine besonderen technischen Schwierigkeiten.

Es ist weiters selbstverständlich, dass die Genauigkeit der Messung desto grösser sein wird, je stärkere Objectivsysteme man benutzt. Für das homogene Immersionssystem Nr. $3=\frac{1}{24}$ " beträgt der Werth eines Scalentheiles $0,541 \mu$. Bei diesem System kann man also, wenn man die Coordinaten einkeilt, selbe bis auf $0,0541 \mu$, d. i. fast $\frac{1}{19000} \mathrm{~mm}$, genau messen.

Die Messung der Z-Coordinate wird durch die Mikrometerschraube des Mikroskops ausgeführt. Zu diesem Zwecke ist der Kopf der erwähnten Schraube in hundert Theile eingetheilt, und da die Ganghöhe dieser Schrauben gewöhnlich ein halb oder ein drittel Millimeter beträgt, so ist theoretisch die Möglichkeit geboten, die $Z$-Coordinate bis auf 0,005 , eventuell 0,003 genau zu messen.

In der Wirklichkeit hängt aber die Genauigkeit dieser Messung von mehreren Umständen ab und zwar :

1) Von der Vollkommenheit der Correction und der Kürze der Brennweite des benutzten Objectivsystems.

Bei einer noch so feinen Theilung des Kopfes der Mikrometerschraube, z. B. in tausend Theile, Anbringung eines Nonius daneben, und bei der 
Voraussetzung einer vollkommenen Genauigkeit der Schraube selbst, wird für die Genauigkeit der Messung noch absolut nichts gewonnen, weil die Unsicherheit der möglichst scharfen Einstellung bei einem schwachen und nicht vollkommen corrigirten Objectivsysleme eine viel zu grosse ist, um die durch eine Drehung der Mikrometerschraube um 10-20, ja vielleicht 100 Theilstriche hervorgebrachte Hebung oder Senkung des Mikroskops mit Sicherheit zu bemerken.

2) Von der Sicherheit der Führung und der Unbeweglichkeit des oberen Mikroskopkörpers (Stärke der Druck- und Hebefeder), damit der Tubus in jede gewünschte Lage gebracht werden kann und in derselben wäbrend der ganzen Beobachtung unbeweglich verbleibt.

3) Von der Genauigkeit der Ausfúhrung der Mikrometerschraube und ihrer Kopftheilung und der Präcision, nit welcher die Höhe ihres Ganges bestimmt ist.

Da aber die durch die erwähnte Schraube gewonnenen Maasse: mit den durch die Scala gewonnenen congruiren sollen, muss das Objectmikrometer auf derselben 'Theilmaschine gemacht oder wenigstens verglichen werden, auf welcher die Höhe des Mikrometerschraubenganges bestimmt wird.

Bei der Erfüllung aller dieser Bedingungen wird der direct gefundene Werth der Z-Coordinate nur dann der Wahrheit entsprechen, wenn das gemessene Object und das Linsensystem in demselben, oder in optisch gleichwerthigen Medien eintauchen. In jedem anderen Fall muss man den direct gefundenen Werth reduciren, indem man ihn mit dem Verhältniss der Brechungsindices dieser Medien multiplicirt.

Gesetzt z. B., dass für einen Punkt eines in Balsam eingeschlossenen Krystalls die $Z$-Coordinate mit einem Trockenobjectivsystem $45,3 \mu$, mit einem Wasserimmersionssystem $60,2 \mu$ und mit einem homogenen Oelimmersionssystem $68 \mu$ betrug, so kann nur diese letzte Zahl, weil Cedernholzöl und Balsam optisch gleichartig sind, der Wahrheit entsprechen. Um die zwei anderen direct gefundenen Zahlen zu corrigiren, braucht man sie nur, da die entsprechenden Indices 1, 1,33 und 1,5 gleich sind, mit ihren Verhältnissen zu multipiciren und zwar wird für das trockene System $45,3 \times \frac{1,5}{1}=68,05$, für das Wasserimmersionssystem $60,2 \times \frac{1,5}{1,33}=$ 67,8699 , also fast gänzlich übereinstimmende Zahlen.

Bei der Anwendung der besten, vollkommen corrigirten homogenen Immersionssysteme und der Erfüllung aller oben genannten Bedingungen lässt sich der unvermeidliche Einstellungsfehler sicher unter $1 \mu=0,001 \mathrm{~mm}$ herabdrucken.

Um die Principien und den Gang der Berechnung zu zeigen, bezeichnen wir die drei Punkte, die auf einer der beiden Ebenen liegen, mit 1, 2 und 3 und ihre entsprechenden Coordinaten mit $X_{1} Y_{1} Z_{1}, X_{2} Y_{2} Z_{2}, X_{3} Y_{3} Z_{3}$; die 
drei Punkle, welche auf der zweiten Ebene liegen, mit 4, 5 und 6 und ihre entsprechenden Coordinaten mit $X_{4} Y_{4} Z_{4}, X_{5} Y_{5} Z_{5}, X_{6} Y_{6} Z_{6}$; die auf dieselben rechtwinkligen Goordinaten bezogene Gleichung der ersten Ebene mit

$$
A x+B y+C z=D
$$

und endlich der zweiten mit

$$
A_{1} x+B_{1} y+C_{1} z=D_{1} \text {. }
$$

Da die Punkte 1, 2 und 3 auf der ersten und die Punkte 4, 丂̆ und 6 auf der zweiten Ebene liegen, so mussen die Coordinaten dieser Punkte den Gleichungen dieser Ebenen gentigen. Es müssen also gleichzeitig folgende Gruppen von Gleichungen stattfinden:

$$
\left.\begin{array}{l}
A x_{1}+B y_{1}+C z_{1}=D \\
A x_{2}+B y_{2}+C z_{2}=D \\
A x_{3}+B y_{3}+C z_{3}=D
\end{array}\right\}
$$

und

$$
\left.\begin{array}{l}
A_{1} x_{4}+B_{1} y_{4}+C_{1} z_{4}=D_{1} \\
A_{1} x_{5}+B_{1} y_{5}+C_{1} z_{5}=D_{1} \\
A_{1} x_{6}+B_{1} y_{6}+C_{1} z_{6}=D_{1}
\end{array}\right\},
$$

aus denen man durch Elimination die Werthe der Coëfficienten $A, B, C$, $A_{1}, B_{1}, C_{1}$ bestimmen kann.

Und zwar ist :

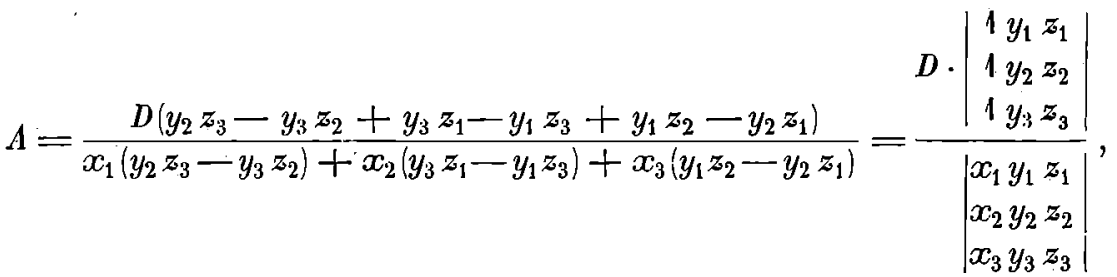

$$
\begin{aligned}
& \boldsymbol{B}=\frac{D\left(x_{3} z_{2}-x_{2} z_{3}+x_{1} z_{3}-x_{3} z_{1}+x_{2} z_{1}-x_{1} z_{2}\right)}{x_{1}\left(y_{2} z_{3}-y_{3} z_{2}\right)+x_{2}\left(y_{3} z_{1}-y_{1} z_{3}\right)+x_{3}\left(y_{1} z_{2}-y_{2} z_{1}\right)}=\frac{D:\left|\begin{array}{lll}
x_{1} & 1 & z_{1} \\
x_{2} & 1 & z_{2} \\
x_{3} & 1 & z_{3}
\end{array}\right|}{\left|\begin{array}{l}
x_{1} y_{1} z_{1} \\
x_{2} y_{2} z_{2} \\
x_{3} y_{3} z_{3}
\end{array}\right|}, \\
& C=\frac{D\left(x_{2} y_{3}-x_{3} y_{2}+x_{3} y_{1}-x_{1} y_{3}+x_{1} y_{2}-x_{2} y_{1}\right)}{x_{1}\left(y_{2} z_{3}-y_{3} z_{2}\right)+x_{2}\left(y_{3} z_{1}-y_{1} z_{3}\right)+x_{3}\left(y_{1} z_{2}-y_{2} z_{1}\right)}=\frac{\text { D. }\left|\begin{array}{lll}
x_{1} & y_{1} & 1 \\
x_{2} & y_{2} & 1 \\
x_{3} & y_{3} & 1
\end{array}\right|}{\left|\begin{array}{l}
x_{1} y_{1} z_{1} \\
x_{2} y_{2} z_{2} \\
x_{3} y_{3} z_{3}
\end{array}\right|},
\end{aligned}
$$




$$
\begin{aligned}
& A_{1}=\frac{D_{1}\left(y_{5} z_{6}-y_{6} z_{5}+y_{6} z_{4}-y_{4} z_{6}+y_{4} z_{5}-y_{5} z_{4}\right)}{x_{4}\left(y_{5} z_{6}-y_{6} z_{5}\right)+x_{5}\left(y_{6} z_{4}-y_{4} z_{6}\right)+x_{6}\left(y_{4} z_{5}-y_{5} z_{4}\right)}=\frac{D_{1} \cdot\left|\begin{array}{ll}
1 & y_{4} z_{4} \\
1 & y_{5} z_{5} \\
1 & y_{6} z_{6}
\end{array}\right|}{\left|\begin{array}{l}
x_{4} y_{4} z_{4} \\
x_{5} y_{5} z_{5} \\
x_{6} y_{6} z_{6}
\end{array}\right|}, \\
& B_{1}=\frac{D_{1}\left(x_{6} z_{5}-x_{5} z_{6}+x_{4} z_{6}-x_{6} z_{4}+x_{5} z_{4}-x_{4} z_{5}\right)}{x_{4}\left(y_{5} z_{6}-y_{6} z_{5}\right)+x_{5}\left(y_{6} z_{4}-y_{4} z_{6}\right)+x_{6}\left(y_{4} z_{5}-y_{5} z_{4}\right)}=\frac{D_{1} \cdot\left|\begin{array}{lll}
x_{4} & 1 & z_{4} \\
x_{5} & 1 & z_{5} \\
x_{6} & 1 & z_{6}
\end{array}\right|}{\left|\begin{array}{l}
x_{4} y_{4} z_{4} \\
x_{5} y_{5} z_{5} \\
x_{6} y_{6} z_{6}
\end{array}\right|}, \\
& C_{1}=\frac{D_{1}\left(x_{5} y_{6}-x_{6} y_{5}+x_{6} y_{4}-x_{4} y_{6}+x_{4} y_{5}-x_{5} y_{4}\right)}{x_{4}\left(y_{5} z_{6}-y_{6} z_{5}\right)+x_{5}\left(y_{6} z_{4}-y_{4} z_{6}\right)+x_{6}\left(y_{4} z_{5}-y_{5} z_{4}\right)}=\frac{D_{1} \cdot\left|\begin{array}{l}
x_{4} y_{4} 1 \\
x_{5} y_{5} 1 \\
x_{6} y_{6} 1
\end{array}\right|}{\left|\begin{array}{l}
x_{4} y_{4} z_{4} \\
x_{5} y_{5} z_{5} \\
x_{6} y_{6} z_{6}
\end{array}\right|},
\end{aligned}
$$

und wenn wir noch zur Abkürzung die Determinanten

$$
\begin{aligned}
& \left|\begin{array}{lll}
1 & y_{1} & z_{1} \\
1 & y_{2} & z_{2} \\
1 & y_{3} & z_{3}
\end{array}\right|,\left|\begin{array}{lll}
x_{1} & 1 & z_{1} \\
x_{2} & 1 & z_{2} \\
x_{3} & 1 & z_{3}
\end{array}\right|,, \\
& \left|\begin{array}{lll}
1 & y_{4} & z_{4} \\
1 & y_{5} & z_{5} \\
1 & y_{6} & z_{6}
\end{array}\right|, \quad\left|\begin{array}{lll}
x_{1} & y_{1} & 1 \\
x_{2} & y_{2} & 1 \\
x_{3} & y_{3} & 1
\end{array}\right|,
\end{aligned}
$$

der Reihe nach mit:

bezeichnen, ist:

$$
\begin{aligned}
& a, b, c, \Delta \\
& a_{1}, b_{1}, c_{1}, \Delta_{1}
\end{aligned}
$$

$$
\begin{aligned}
& A=\frac{D \cdot a}{d}, \quad B=\frac{D \cdot b}{d}, \quad C=\frac{D \cdot c}{d}, \\
& A_{1}=\frac{D_{1} \cdot a_{1}}{A_{1}}, \quad B_{1}=\frac{D_{1} \cdot b_{1}}{A_{1}}, \quad C_{1}=\frac{D_{1} \cdot c_{1}}{\Delta_{1}} \text {. }
\end{aligned}
$$

Wenn wir den Winkel zwischen den beiden Ebenen mit $\lambda$ bezeichnen, so ist, da die Gleichungen dieser Ebenen auf rechtwinklige Coordinaten bezogen sind:

$$
\cos \lambda=\frac{A A_{1}+B B_{1}+C C_{1}}{\sqrt{A^{2}+B^{2}+C^{2}} \cdot \sqrt{A_{1}^{2}+B_{1}^{2}+C_{1}^{2}}}
$$


und wenn wir in diese Formel die durch die Gleichungen (5) und (6) gegebenen Werthe der Coëficienten einsetzen, so.ist:

$$
\begin{aligned}
& \cos \lambda=\frac{\frac{D a}{\Delta} \cdot \frac{D_{1} a_{1}}{\Delta_{1}}+\frac{D b}{\Delta} \cdot \frac{D_{1} b_{1}}{\Delta_{1}}+\frac{D c}{\Delta} \cdot \frac{D_{1} c_{1}}{\Delta_{1}}}{\sqrt{\frac{D^{2} a^{2}}{\Delta^{2}}+\frac{D^{2} b^{2}}{\Delta^{2}}+\frac{D^{2} c^{2}}{\Delta^{2}}} \cdot \sqrt{\frac{D_{1}^{2} a_{1}^{2}}{\Delta_{1}^{2}}+\frac{D_{1}^{2} b_{1}{ }^{2}}{\Delta_{1}{ }^{2}}+\frac{D_{1}{ }^{2} c_{1}{ }^{2}}{\Delta_{1}{ }^{2}}}} \\
& =\frac{\frac{D \cdot D_{1}}{d A_{1}}\left(a a_{1}+b b_{1}+c c_{1}\right)}{\frac{D \cdot D_{1}}{\Delta \Delta_{1}} \sqrt{a^{2}+b^{2}+c^{2}} \cdot \sqrt{a_{1}^{2}+b_{1}^{2}+c_{1}^{2}}}
\end{aligned}
$$

und endlich nach der Elimination des gemeinschaftlichen Factors

$$
\cos \lambda=\frac{a a_{1}+b b_{1}+c c_{1}}{\sqrt{a^{2}+b^{2}+c^{2}} \cdot \sqrt{a_{1}^{2}}+b_{1}^{2}+c_{1}^{2}},
$$

wo

$$
\left.\begin{array}{r}
a=y_{2} z_{3}-y_{3} z_{2}+y_{3} z_{1}-y_{1} z_{3}+y_{1} z_{2}-y_{2} z_{1} \\
b=x_{3} z_{2}-x_{2} z_{3}+x_{1} z_{3}-x_{3} z_{1}+x_{2} z_{1}-x_{1} z_{2} \\
c=x_{2} y_{3}-x_{3} y_{2}+x_{3} y_{1}-x_{1} y_{3}+x_{1} y_{2}-x_{2} y_{1} \\
a_{1}=y_{5} z_{6}-y_{6} z_{5}+y_{6} z_{4}-y_{4} z_{6}+y_{4} z_{5}-y_{5} z_{4} \\
b_{1}=x_{6} z_{5}-x_{5} z_{6}+x_{4} z_{6}-x_{6} z_{4}+x_{5} z_{4}-x_{4} z_{5} \\
c_{1}=x_{5} y_{6}-x_{6} y_{5}+x_{6} y_{4}-x_{4} y_{6}+x_{4} y_{5}-x_{5} y_{4}
\end{array}\right\} .
$$

Um also einen diëdrischen Winkel eines mikroskopischen Krystalles zu bestimmen, genugt es, nachdem die Coordinaten von sechs Punkten auf die beschriebene Weise ausgemessen werden, selhe in die Gleichungen (8) einzusetzen, aus diesen einfachen Formeln die Grössen $a, b, c, a_{1}, b_{1}, c_{1}$ auszurechnen und diese letzten in die Formel (7) einzusetzen.

Wenn die Durchschnittskante der beiden Krystallfächen, zwischen welchen man den diëdrischen Winkel bestimmen will, ausgebildet wäre, und man auf derselben zwei Punkte wählen könnte, wären diese Punkte für die beiden Ebenen gemein, und man hätte in diesem Falle auf jeder dieser Ebenen nur noch einen einzigen Punkt zu wählen, also im Ganzen die Coordinaten von nur vier Punkten zu bestimmen.

Wenn eine der beiden Krystallflächen, z. B. die zweite

$$
A_{1} x+B_{1} y+C_{1} z=D_{1}
$$

der $X Y$-Ebene (der Tischebene) parallel wäre, $d$. h. wenn

$$
z_{4}=z_{5}=z_{6}=\gamma,
$$

so wäre, wie man sich durch Substitution leicht überzeugen kann :

$$
a_{1}=b_{1}=0 \text {, }
$$


und somit

oder endlich

$$
\cos \lambda=\frac{c c_{1}}{\sqrt{a^{2}+b^{2}+c^{2}} \cdot \sqrt{c_{1}^{2}}}=\frac{c c_{1}}{c_{1} \sqrt{a^{2}+b^{2}+c^{2}}}
$$

$$
\cos \lambda=\frac{c}{\sqrt{a^{2}+b^{2}+c^{2}}}
$$

eine, im Vergleich zu der vorigen (7), viel einfachere Formel, zu welcher man unmittelbar gelangen kann; denn wenn die zweite Krystallfläche zu der $X Y$-Ebene parallel ist, so ist der Winkel zwischen den beiden Ebenen gleich dem Winkel zwischen der ersten und der X $Y$-Ebene. Dieser Winkel aber ist dem Winkel zwischen der zu der ersten Ebene Senkrechten (Normalen) und der Z-Axe gleich, und der Cosinus dieses letzten Winkels ist, wie bekannt, wenn die Gleichung der Ebene

$$
A x+B y+C z=D,
$$

durch die Formel gegeben

$$
\cos \lambda=\frac{C}{\sqrt{A^{2}+B^{2}+C^{2}}} .
$$

Setzt man aber in diese Formel die oben (5) gefundenen Werthe fur $A, B$ und $C$ und eliminirt zwischen den Zähler und Nenner den gemeinschaftlichen Factor $\frac{D}{A}$, so kommt man unmittelbar auf die Formel (9).

Wenn, im noch specielleren Falle, nicht nur die zweite Ebene zu der X Y-Ebene parallel wäre, sondern noch die Durchschnittskante der beiden Ebenen ausgebildet wäre und zum Parallelelimus mit einer der Axen, z. B. der $X$-Axe gebracht werden könnte, und man auf dieser Kante zwei für beide Ebenen gemeinschaftliche Punkte wählen würde, d. h. wenn

$$
\begin{aligned}
& z_{1}=z_{2}=z_{4}=z_{5}=z_{6}=\gamma \\
& y_{1}=y_{2}=\beta,
\end{aligned}
$$

so wäre, wenn man diese Werthe in die Formel (8) einsetzl:

$$
\begin{aligned}
& a=\beta \gamma-y_{3} \gamma+y_{3} \gamma-\beta \gamma+\beta \gamma-\beta \gamma=0, \\
& b=x_{3} \gamma-x_{2} z_{3}+x_{1} z_{3}-x_{3} \gamma+x_{2} \gamma-x_{1} \gamma=z_{3}\left(x_{1}-x_{2}\right)-\gamma\left(x_{1}-x_{2}\right) \\
&=\left(z_{3}-\gamma\right)\left(x_{1}-x_{2}\right), \\
& c=x_{2} y_{3}-x_{3} \beta+x_{3} \beta-x_{1} y_{3}+x_{1} \beta-x_{2} \beta=\beta\left(x_{1}-x_{2}\right)-y_{3}\left(x_{1}-x_{2}\right) \\
&=\left(\beta-y_{3}\right)\left(x_{1}-x_{2}\right),
\end{aligned}
$$

und wenn man diese Werthe für $a, b$ und $c$ in die Formel (9) einsetzt, so ist

$$
\cos \lambda=\frac{\left(x_{1}-x_{2}\right)\left(\beta-y_{3}\right)}{\sqrt{\left.x_{1}-x_{2}\right)^{2}\left(z_{3}-\gamma\right)^{2}+\left(x_{1}-x_{2}\right)^{2}\left(\beta-y_{3}\right)^{2}}}
$$




$$
=\frac{\left(x_{1}-x_{2}\right)\left(\beta-y_{3}\right)}{\left(x_{1}-x_{2}\right) \cdot \sqrt{\left(z_{3}-\gamma\right)^{2}+\left(\beta-y_{3}\right)^{2}}},
$$

also endlich

$$
\cos \lambda=\frac{\beta-y_{3}}{\sqrt{\left(z_{3}-\gamma\right)^{2}+\left(\beta-y_{3}\right)^{2}}}
$$

somit also

und

$$
\sin \lambda=\frac{z_{3}-\gamma}{\sqrt{\left(z_{3}-\gamma\right)^{2}+\left(\beta-\bar{y}_{3}\right)^{2}}}
$$

$$
\operatorname{tg} \lambda=\frac{z_{3}-\gamma}{\beta-y_{3}}
$$

eine ausserordentlich einfache Formel, zu der man jedoch viel leichter aus der unmittelbaren Betrachtung einer entsprechenden Figur kommen kann.

Wenn endlich die $X Y$ parallele Ebene mit der $X Y$-Ebene selbst, und die parallele Kante mit der $X$-Axe selbst coincidiren würde, das ist, wenn bei allen vorigen Bedingungen noch

$$
\begin{gathered}
\gamma=0 \text { und } \beta=0 \text { wäre, so wäre } \\
\operatorname{tg} \lambda=-\frac{z_{3}}{y_{3}} .
\end{gathered}
$$

Die detaillirte Discussion der oben gewonnenen Formeln (7), (9), (11) und (12) und der Bedeutung ihrer Vorzeichen glaube ich ubergehen zu dürfen, will jedoch ausdrucklich betonen, dass die Coordinaten, wie uberhaupt bei jeder analytischen Aufgabe, so auch hier nicht nur ihrer absoluten Grösse, sondern auch ihren Vorzeichen nach bestimmt werden müssen.

J. T houlet in seiner Abbandlung*) giebt als eine Verbesserung der Werthe i m'schen eine graphische Methode der Winkelbestimmung an. Er verfährt dabei ungefähr folgendermassen :

Man wäblt auf der Kante der beiden Flächen zwei leicht wieder zu erkennende Punkte, auf jeder Fläche einen weiteren Punkt, stellt successive bei unveränderter Lage des Krystalls auf jeden der vier Punkte ein, und liest an der Mikrometerschraube des Tubus ihre Höbendifferenzen ab. Hierauf ersetzt man, ohne das Präparat irgendwie zu verschieben, 'das Ocular durch eine Camera lucida, zeichnet den Krystall durch diese ab, markirt die ihrer Höhenlage nach bestimmten vier Punkte durch feine Nadelstiche und verbindet dieselben durch gerade Linien. Nun ersetzt man das Präparat durch ein Objectmikrometer und zeichnet die Scala desselben gleichfalls

*) Contribution à l'étude des propriétés physiques des minéraux microscopiques. Annales de Chimie et de Physique cinquième série, Tome XX. Paris 1880, pag. 362. Vergl. auch diese Zeitschr. 4, 222. 
ab. Man hat alsdann die horizontale Projection des Tetraëders der vier Punkte. Die scheinbare Länge der Kanten desselben lässt sich solort vermittelst des durch das Objectmikrometer erhaltenen Maassstabes bestimmen; die scheinbare Länge jeder Kante und die Höhendifferenz ihrer Endpunkte bilden die Katheten rechtwinkliger Dreiecke, deren Hypotenusen die wirkliche Länge der Tetraëderkantén darstellen. Aus der bekannten Länge der drei Seiten der drei in einem Tetraëdereck zusammenstossenden geradlinigen Dreiecke berechnet man nach bekannter Formel die drei in dem Eck zusammenstossenden Winkel derselben und hat damit die drei Seiten eines sphärischen Dreiecks, aus dem ein Winkel (der Raumwinkel) zu berechnen ist.

Diese graphische Methode durfte keine grosse Genauigkeit bieten. Wollte man aber, wie es Thoulet (s. dies. Zeitschr. a. a. O.) ebenfalls angiebt, mit dem Ocularmikrometer und der Tubusmikrometerschraube die senkrechten Coordinaten der vier Tetraëderspitzen bestimmen, und aus diesen Gegebenen den gesuchten Winkel ausrechnen, so würde dies folgendermassen zu gescbehen sein.

Wenn wir die zwei auf der Schnittkante der beiden Ebenen liegenden Punkte mit 1 und 2, den auf der ersten Ebene liegenden mit 3 und den aúf der zweiten mit 4 , ihre Coordinaten aber entsprechend mit $x_{1} y_{1} z_{1}, x_{2} y_{2}$ $z_{2}, x_{3} y_{3} z_{3}$ und $x_{4} y_{4} z_{4}$ bezeichnen, so isl nach der bekannten Formel für die Entfernung zweier Punkte im Raume:

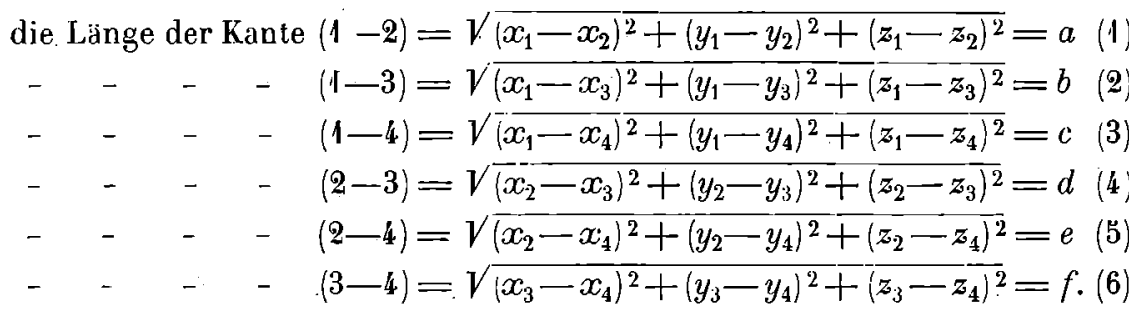

Aus der bekannten Länge der Kanten lassen sich leicht die dreieckigen Flächen des Tetraëders ausrechnen, und zwar ist:

$$
\begin{aligned}
\text { die Fl. d.Dreiecks }(213) & =\frac{1}{4} \sqrt{(a+b+d)(a+b-d)(a+d-b)}(\overline{b+d-a}) \\
--\quad-\quad & (214)=\frac{1}{4} \sqrt{(a+c+e)(a+c-e)(a+e-c)}(\overline{c+e-a)} \\
--\quad-\quad(314) & =\frac{1}{4} \sqrt{(b+c+f)(b+c-f)(b+f-c)(c+f-b)}
\end{aligned}
$$

da aber die Fläche eines beliebigen Dreiecks

$$
\triangle=\frac{1}{2} m n \sin \delta
$$

wo $m$ und $n$ die Längen zweier anstossenden Seiten und $\delta$ die Grösse des zwischen ihnen liegenden Winkels bedeutet, so ist auch : 
L'eber ein neues Ocularmikrom. u. dessen Anwend. in der mikrosk. Krystallogr. 31

$$
\begin{aligned}
\text { die Fläche des Dreiecks }(213) & =\frac{1}{2} a b \sin (213) \\
-\quad-\quad-\quad & (214)=\frac{1}{2} a c \sin (214) \\
-\quad-\quad-\quad(314) & =\frac{1}{2} b c \sin (314),
\end{aligned}
$$

und aus der Combination dieser letzten drei Formeln mit den vorbergehenden (7), (8) und (9) erhalten wir die Werthe der Sinus der im Tetraëderspilz $\uparrow$ zusammenstossenden drei Flächenwinkel, und zwar ist:

$$
\begin{aligned}
& \sin (213)=\frac{\sqrt{(a+b+d)(a+b-d)(a+d-b)(b+d-a)}}{2 a b} \\
& \sin (214)=\frac{\sqrt{(a+c+e)(a+c-e)(a+e-c)(c+e-a)}}{2 a c} \\
& \sin (314)=\frac{\sqrt{(b+c+f)(b+c-f)(b+f-c)(c+f-b)}}{2 b c} .
\end{aligned}
$$

Wenn wir weiter die diesen Winkeln entsprechenden Bögen mit $\alpha, \beta$ und $\gamma$ bezeichnen, und selbe als die Seiten eines aus der Tetraëderspitze 1 als Mittelpunkt mil dem Radius $=1$ gezogenen sphärischen Dreiecks betrachten, so können wir aus der bekannten Länge der Seiten dieses spbärischen Dreiecks die Grösse seiner Winkel bestimmen, und zwar wenn wir noch

selzen, ist

$$
\alpha+\beta+\gamma=2 s
$$

$$
\operatorname{tg} \frac{1}{2} C=\sqrt{\frac{\sin (s-\alpha) \cdot \sin (s-\beta)}{\sin s \cdot \sin (s-\gamma)}},
$$

wo $C$ den sphärischen Winkel zwischen den Bögen $\alpha$ und $\beta$, also auch gleichzeitig den gesuchten diëdrischen Winkel zwischen den Tetraëderflächen (213) und (214) bedeutet.

Wie aus dem Obigen ersichtlich, ist diese letzte Methode unvergleichlich mühsamer und complicirter als die erste, da man bei ihrer Anwendung dreizehn ziemlich complicirte Formeln ausrechnen muss, um den gesuchten diëdrischen Winkel zu bestimmen, während bei der ersten im ungünstigsten Falle sieben viel einfachere Formeln zum Ziele führen.

Das beschriebene Instrument eignet sich endlich vorzüglich zur genauen und bequemen Messung der planen Winkel.

$\mathrm{Zu}$ diesem Zwecke genügt es, wo immer der Winkel am Sehfeld liegen würde, durch entsprechende Drehung des oberen Oculartheils und der an der Trommel angebrachten Schrauben, die lange Kathete der Scala zur gänzlichen Coincidenz mit einem Winkelarm zu bringen und an der Trommelperipherie den Stand des Nonius abzulesen, dann durch weitere Drehung 
32 S. Krysiński. Ueber ein neues Ocularmikrom. und dessen Anwendung etc.

und Verschiebung dieselbe Kathete zur Coincidenz mit dem zweiten Winkelarm zu bringen und den Nonius abermals abzulesen. Die algebraische Differenz beider abgelesenen Werthe giebt unmittelbar das Maass des gesuchten Winkels bis auf 3 Bogenminuten genau an.

Die Vorzuge der beschriebenen Construction lassen sich dahin präcisiren, dass man mit diesem verhältnissmässig wenig complicirten und billigen Instrument bei jedem guten Mikroskopstativ die Messung der linearen Grössen, der planen und der diëdrischen Winkel viel leichter und genauer ausfuhren kann, als mit allen anderen noch so complicirten Instrumenten und speciellen Stativen. 